\title{
Optimum ratio of electron-to-hole mobility in P3HT:PCBM organic solar cells
}

\author{
Omar Ramírez, Víctor Cabrera ${ }^{*}$ and Luis Martín Reséndiz \\ Sección de Estudios de Postgrado e Investigación \\ UPIITA, Instituto Politécnico Nacional \\ Av. IPN 2580, 07340 D.F., México \\ vcabreraa@ipn.mx
}

\begin{abstract}
We investigate the influence of different electron-to-hole mobility ratios on the performance of a P3HT:PCBM bulk heterojunction organic solar cell by numerical simulations. We show that using a ratio between electron and hole mobility higher than one, in some particular mobility cases, it can lead to an improvement on the power conversion efficiency.
\end{abstract}

Keywords-organic semiconductors; solar cells; charge carrier mobility; numerial simulations; efficiency

\section{INTRODUCTION}

Organic solar cells have shown an important improvement based on intensive research and development in the last decade. The currently most efficient class of polymer-based solar cells are based on the bulk heterojunction concept, which consists of a blend of donor and acceptor materials mixed in a solution forming two interpenetrating networks. Bulk heterojunction solar cell based on the blend of poly (3-hexylthiophene) (P3HT) and 6,6-phenyl C61-butyric acid methyl ester (PCBM) is currently one of the most promising organic photovoltaic devices due to efficient dissociation of excitons and high carrier mobility.

Recently, several studies focus on the development of more efficient organic solar devices had led to the research of charge carrier mobility due to the fact that in order to improve the power conversion efficiency, it is necessary to collect as many photogenerated carriers as possible in the electrodes of the solar cell. The collection of charge carriers depends on their mobility and recombination.

We show that short circuit current density does not vary with different electron-to-hole mobility ratios while the open circuit voltage decreases. However, we have found that the maximum power point of the solar cell can be improved by using an optimum electron-to-hole mobility ratio.

\section{MODELS AND METHODS}

Since the electrical model of organic solar cells used in this article has already been explained in great detail in [1], it is partially described in this section. In this research, we have solved numerically the drift-diffusion equations for Langevin recombination model which has been demonstrated as a powerful tool to explain the influence of various effects on the solar cell current-voltage characteristics [1-2]. By using Langevin theory, the bimolecular recombination of charge carriers is given by

$$
R(x)=\gamma\left[n(x) p(x)-n^{2}{ }_{i n t}\right]
$$

where $\gamma$ is the recombination constant determined by the minimum mobility as

$$
\gamma=q / \varepsilon \cdot \min \left(\mu_{n}, \mu_{p}\right)
$$

where $\mu_{n}$ is the electron mobility and $\mu_{p}$ the hole mobility. We define the electron-to-hole mobility ratio $\beta$ as

$$
\beta=\mu_{n} / \mu_{p}
$$

\section{RESULTS AND DISCUSSION}

For this study, we used the same physical parameters of an organic solar cell reported in [3], which corresponds to $\mathrm{BHJ}$ solar cells consisting of $\mathrm{P} 3 \mathrm{HT}$ as the hole conductor and the fullerene derivative PCBM as the electron conductor. The physical parameters used in the simulation are listed in Table I.

In order to investigate the effects of $\beta$ on the electrical properties of the solar cell, we vary the hole mobility from $1 \times 10^{-8} \mathrm{~cm}^{2} /$ Vs to $1 \times 10^{8} \mathrm{~cm}^{2} /$ Vs and simulate four cases: $\beta=$ $1, \beta=10, \beta=100$ and $\beta=1000$.

TABLE I

PARAMETERS USED TO OBTAIN THE SIMULATION RESULTS

\begin{tabular}{lll}
\hline \hline Parameter & Symbol & Numerical value \\
\hline Band gap & $E_{g}$ & $1.1 \mathrm{eV}$ \\
Eff. Density of states & $N_{e f f}$ & $2.5 \times 10^{19} \mathrm{~cm}^{-3}$ \\
Generation rate & $G$ & $9.7 \times 10^{21} \mathrm{~cm}^{-3} \mathrm{~s}^{-1}$ \\
Dielectric constant & $\varepsilon$ & 3.4 \\
e-h pair distance & $a$ & $1.12 \mathrm{~nm}$ \\
Decay rate & $k_{f}$ & $3 \times 10^{5} \mathrm{~s}^{-1}$ \\
Active layer thickness & $d$ & $100 \mathrm{~nm}$ \\
\hline \hline
\end{tabular}

As part of the results of this research, we have found that short circuit current density of the solar cell does not show any significant change of magnitude with higher ratios. As the recombination constant of the Langevin recombination 
model only depends on the lower mobility and the hole mobility was always the lowest one in this case of study, a strong relation between recombination process and short circuit current density can be glimpsed, in other words, the main process involved in determining the short circuit current density is the recombination. Another point to highlight related with short circuit current density is the fact that for mobilities around $1 \times 10^{2} \mathrm{~cm}^{2} / \mathrm{Vs}$ and higher, the magnitude seems to remain as a constant, supporting the theory of the optimum charge carrier mobility. The graph showing these results is shown in Fig. 1 (a).

As can be seen from Fig. 1 (a), open circuit voltage magnitude seems to decrease according with higher mobilities and ratios, except in the interval $1 \times 10^{-8} \mathrm{~cm}^{2} / \mathrm{Vs}<$ $\mu \mathrm{p}<1 \times 10^{-5} \mathrm{~cm}^{2} / \mathrm{Vs}$ where the magnitude for $\beta=10$ and $\beta=$ 100 is higher than the magnitude for $\beta=1$. This decreasing tendency is strictly related with the way in how the model of the solar cell considers the separation distances of electron and hole, as the assumption of a variable electron-hole pair separation has a considerable influence on the open circuit voltage. Reference [4] delves in a better understanding of this phenomenon.

Although Fig. 1 (a) gives an idea about the electrical properties of the solar cell, we cannot be sure if the power conversion efficiency improves or not with higher electronto-hole mobility ratios since the short circuit current density shows an increment but at the same time the open circuit voltage decreases according with higher mobilities. In order to have a better idea about the power conversion efficiency, we calculate the point of maximum power of the solar cell shown in Fig. 1 (b). At first look, the behavior of the Pmax agrees with recently published studies about the existence of an optimum mobility [5]. As can be appreciated, an optimum ratio can be chosen depending on the lowest mobility. For example, whether the lowest mobility (either the electron or hole) is lower than $1 \mathrm{~cm}^{2} / \mathrm{Vs}$, a higher ratio leads to an improvement of the maximum power point. In the other hand, if the lowest mobility is higher than $1 \mathrm{~cm}^{2} / \mathrm{Vs}$, a ratio of one would be enough to obtain the highest Pmax. In this study, the highest point of maximum power $\left(4.888 \mathrm{~mW} / \mathrm{cm}^{2}\right)$ was calculated with a hole mobility of $4.64 \times 10^{-2} \mathrm{~cm}^{2} / \mathrm{Vs}$ and a ratio $\beta=10$ (Fig. 2)

\section{CONCLUSIONS}

We investigate the effects of four different ratios of electron-to-hole mobility on the short circuit current density, open circuit voltage and maximum power point of a P3HT:PCBM bulk heterojunction organic solar cell by numerical simulations. We show that short circuit current density does not have an important improvement for higher ratios and it can be considered even as a constant. Open circuit voltage shows a decreasing tendency for higher mobility ratios, which is consistent with some other reported studies [4]. We also show that, depending on the magnitude of the lowest mobility, the maximum power point (Pmax) can improve about $30 \%$ for electron-to-hole mobility ratios higher than one.
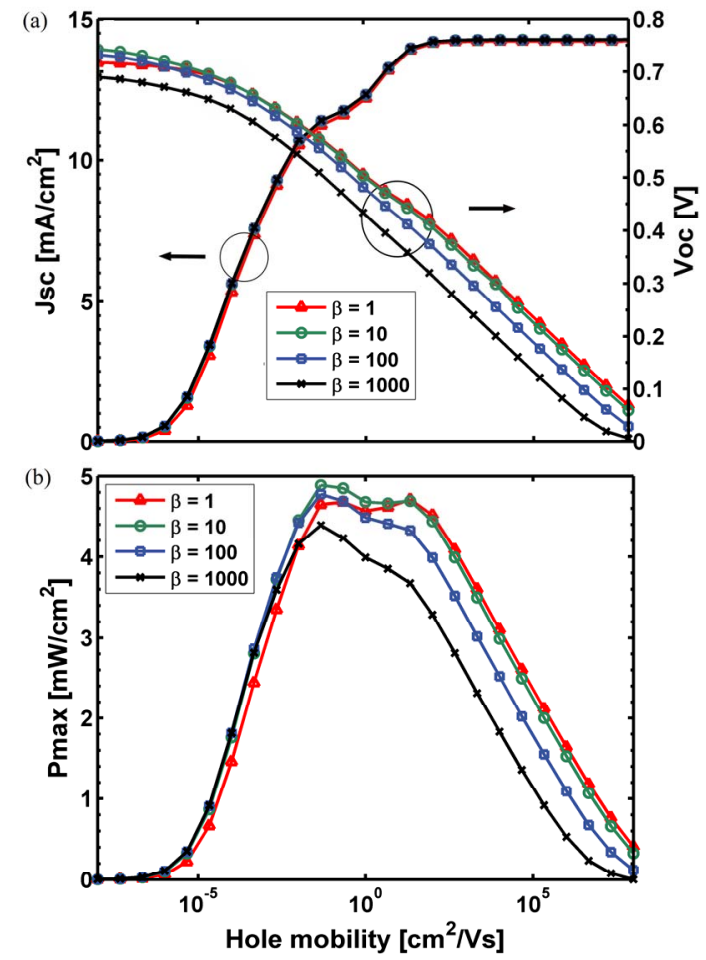

Fig. 1. Short circuit current density, open circuit voltage (a) and maximum power point (b) as a function of the hole mobility for four electron-to-hole mobility ratios.

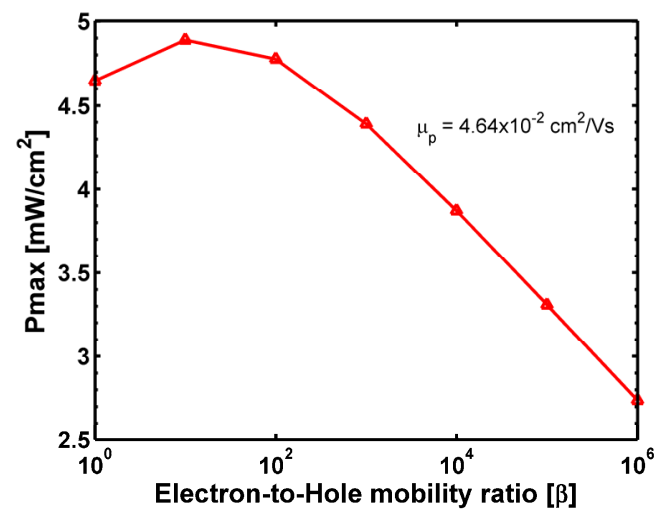

Fig. 2. Maximum power point as a function of the electron-to-hole mobility ratio.

\section{REFERENCES}

[1] L. J. A. Koster, E. . P. Smits, V. D. Mihailetchi, and P. W. M. Blom, "Device model for the operation of polymer/fullerene bulk heterojunction solar cells", Phys. Rev. B 72, 085205 (2005).

[2] W. Tress, K. Leo, and M. Riede, "Influence of hole-transport layers and donor materials on open-circuit voltage and shape of i-v curves of organic solar cells", Adv. Funct. Mater. 21, 2140 (2011).

[3] G. Namkoong, P. Boland, K. Lee, and J. Dean,'Design of organic tandem solar cells using PCPDTBT:PC ${ }_{61} \mathrm{BM}$ and P3HT:PC ${ }_{71} \mathrm{BM}$ ", J. Appl. Phys. 107, 124515 (2010).

[4] T. Kirchartz, B. E. Pieters, K. Taretto, and U. Rau, "Mobility dependet efficiencies of bulk heterojuntion solar cells", Phys. Rev. B 80, 035334 (2009).

[5] W. Tress, K. Leo, and M. Riede, "Omtimum mobility, contact properties, and open-circuit voltage of organic solar cells", Phys. Rev. B 85, 155201 (2012). 